\title{
Study of the $\mathrm{Li}(d, x n)$ reaction for the development of accelerator-based neu- tron sources
}

\author{
Yukinobu Watanabe ${ }^{1, *}$, Hiroki Sadamatsu ${ }^{1}$, Shouhei Araki ${ }^{1}$, Keita Nakano ${ }^{1}$, Shoichiro Kawase ${ }^{1}$, Tadahiro Kin ${ }^{1}$, Yosuke \\ Iwamoto $^{2}$, Daiki Satoh ${ }^{2}$, Masayuki Hagiwara ${ }^{3}$, Hiroshi Yashima ${ }^{4}$, Tatsushi Shima ${ }^{5}$, and Shinsuke Nakayama ${ }^{2}$ \\ ${ }^{1}$ Department of Advanced Energy Engineering Science, Kyushu University, 6-1 Kasuga-koen, Kasuga, Fukuoka 816-8580, Japan \\ ${ }^{2}$ Japan Atomic Energy Agency (JAEA), 2-4 Shirakata, Tokai, Naka, Ibaraki 319-1195, Japan \\ ${ }^{3}$ High Energy Accelerator Research Organization (KEK), 1-1 Oho, Tsukuba, Ibaraki 305-0801, Japan \\ ${ }^{4}$ Research Reactor Institute, Kyoto University, 2-1010 Asashiro-nishi, Kumatori, Sennan, Osaka 590-0494, Japan \\ ${ }^{5}$ Research Center for Nuclear Physics (RCNP), Osaka University, 10-1 Mihogaoka, Ibaraki, Osaka 567-0047, Japan
}

\begin{abstract}
Double-differential neutron production cross sections (DDXs) for deuteron-induced reactions on Li at $200 \mathrm{MeV}$ were measured for emission angles ranging from $0^{\circ}$ to $25^{\circ}$ in steps of $5^{\circ}$ by means of a time of flight (TOF) method with EJ301 liquid organic scintillators at the Research Center for Nuclear Physics (RCNP), Osaka University. The measured DDXs were compared to theoretical model calculations with the DEURACS and PHITS codes and TENDL-2017 nuclear data. It was found that the DEURACS calculation is in better agreement with the measured DDXs than the PHITS calculation, while TENDL-2017 fails to reproduce both the spectral shape and magnitude of the measured DDXs for all angles.
\end{abstract}

\section{Introduction}

Accelerator-based neutron sources utilizing deuteroninduced reactions on $\mathrm{Li}, \mathrm{Be}, \mathrm{C}$, etc., are proposed for various neutron beam applications such as radioisotopes production for medical use, irradiation tests of fusion reactor materials, and transmutation of long-lived radioactive nuclear waste. Neutron production data for neutron converters bombarded by deuterons are required for optimizing the design of such neutron sources. However, the experimental data, e.g. double-differential neutron production cross sections (DDXs), are not sufficient. We pay attention to lithium as one of the promising candidates of neutron converters. Several experimental data of thick target neutron yields (TTNYs) have been reported at incident deuteron energies ranging from 5 to $40 \mathrm{MeV}$ [1], while DDX data measured with thin target are very limited to only three incident energies of $25 \mathrm{MeV}$ [2], $40 \mathrm{MeV}$ [3], and $102 \mathrm{MeV}$ [4]. Compared to TTNYs, DDX data are more useful for direct benchmark tests of theoretical models and evaluated nuclear data.

In the present work, we have conducted a new DDX measurement for $\mathrm{Li}$ at higher incident deuteron energy of $200 \mathrm{MeV}$ in the Research Center for Nuclear Physics (RCNP), Osaka University for further investigating the incident energy dependence of neutron production from thin Li target bombarded by deuterons. Moreover, the experimental data are compared with theoretical model calculations with PHITS (Particle and Heavy Ion Transport System) [5] and DEURACS (Deuteron-induced Re-

*e-mail: watanabe@aees.kyushu-u.ac.jp action Analysis Code System) [6] and TENDL-2017 nuclear data [7] for benchmark tests.

\section{Experimental method}

The experiment was carried out at the N0 course in RCNP. The experimental setup and procedure were almost the same as in our past measurement done at $102 \mathrm{MeV}$ [4].

The experimental setup is schematically shown in Figure 1. A deuteron beam accelerated to $200 \mathrm{MeV}$ was transported to the neutron experimental hall and bombarded a 1-mm thick natural $\mathrm{Li}$ target foil having the chemical purity of $99.9 \%$ which was placed in the beam swinger magnet. Then, the deuterons passing through the target were delivered to a Faraday cup. The beam current was changed from 3 to $45 \mathrm{nA}$, depending on neutron detection angles. Emitted neutrons from the Li target were detected by two different-size EJ301 liquid organic scintil-

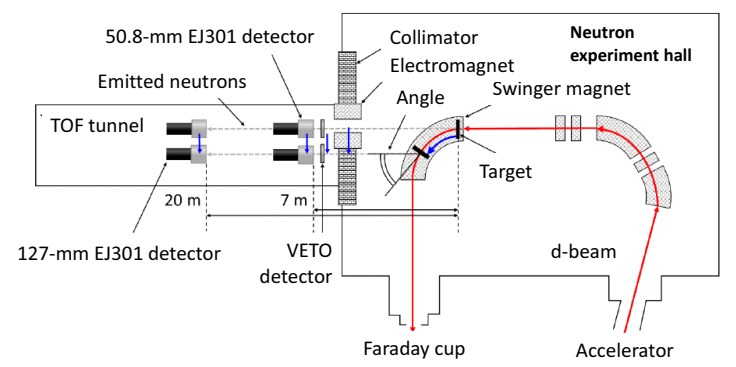

Figure 1. Layout of the experimental setup. 
lators $(50.8 \mathrm{~mm}$ by $50.8 \mathrm{~mm}$ and $127 \mathrm{~mm}$ by $127 \mathrm{~mm}$ in diameter and length, which hereafter will be referred to as 50.8-mm EJ301 detector and 127-mm EJ301 detector, respectively) located at two different distances of $7 \mathrm{~m}$ and 20 $\mathrm{m}$, respectively. The neutron DDXs were measured at six angles $\left(0^{\circ}, 5^{\circ}, 10^{\circ}, 15^{\circ}, 20^{\circ}\right.$, and $\left.25^{\circ}\right)$ by moving the target along the beam trajectory in the swinger magnet. The emitted neutrons were transported to the 100-m TOF tunnel through a movable collimator. The position of the collimator was changed depending on the target position. A clearing magnet was installed in the collimator to remove background components of charged particles.

The energies of emitted neutrons were measured by the conventional TOF method with two different sizes of EJ301 liquid organic scintillators (50.8-mm and 127-mm detectors) as shown in Figure 1. Since the magnetic field of the clearing magnet influenced the photomultiplier tube at $7 \mathrm{~m}$, the magnet was switched off and a $5 \mathrm{~mm}$ thick NE102A plastic scintillator was additionally mounted in front of the 50.8-mm EJ301 detector as a veto detector of background charged particles.

\section{Data analysis}

The data analysis method is the same as in our previous work [4].

The light outputs from the EJ301 detectors were calibrated with two kinds of standard RI sources, ${ }^{137} \mathrm{Cs}$ and ${ }^{241} \mathrm{Am} / \mathrm{Be}$, and the thresholds of the 50.8- mm and $127-\mathrm{mm}$ EJ301 detectors were set to be $0.49 \mathrm{MeVee}$ and $4.3 \mathrm{MeVee}$, respectively. In the energy range lower than $15 \mathrm{MeV}$, the number of background gamma-ray events was comparable to that of neutron events in the measurement with the 50.8mm EJ301 detector. Both the events were well separated by a conventional pulse shape discrimination method.

The prompt gamma peak observed in the measured TOF spectrum was used as a reference point to obtain the absolute neutron flight time. The neutron energy was calculated from the flight time, and then the TOF spectrum was converted to the energy spectrum. The experimental DDX was derived from the energy spectrum using the target thickness, the solid angle subtended by the detector, the deuteron beam current, the neutron detection efficiency, and the attenuation correction of neutron fluxes. The neutron detection efficiencies for the two EJ301 scintillators were calculated with the SCINFULQMD code [8]. The attenuation of neutron fluxes in air at $7 \mathrm{~m}$ and $20 \mathrm{~m}$ was estimated by PHITS calculation with JENDL/HE-2007 [9]. Finally, both the DDXs measured at $7 \mathrm{~m}$ and $20 \mathrm{~m}$ were merged at $12 \mathrm{MeV}$. Accordingly, each experimental DDX shown in Figure 2 is composed of the DDX measured at $7 \mathrm{~m}$ in the energy range less than 12 $\mathrm{MeV}$ and that measured at $20 \mathrm{~m}$ above $12 \mathrm{MeV}$.

Uncertainties of the measurements are due to systematic and statistical errors. The systematic error was estimated to be $12 \%$, and the major causes come from the detection efficiencies calculated by the SCINFUL-QMD code $(10 \%)$, the solid angle $(<1 \%)$, the attenuation correction in air estimated by the PHITS calculation $(3 \%)$,

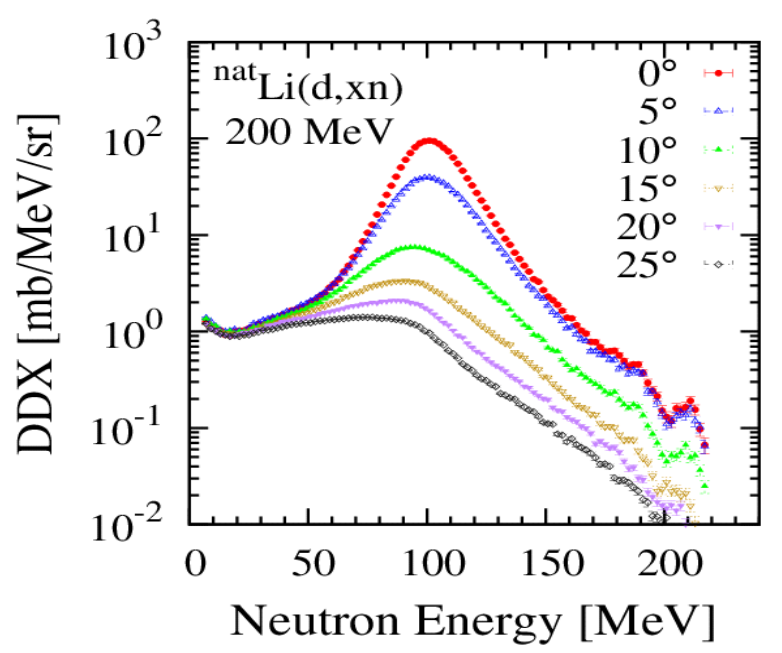

Figure 2. Measured double-differential cross sections (DDXs) of the $(d, x n)$ reaction on $\mathrm{Li}$ at an incident energy of $200 \mathrm{MeV}$.

and the effect of neutron scattering from the swinger magnet, the floor, the wall, and the collimator $(6 \%)$.

\section{Results and discussion}

\subsection{Experimental results}

Figure 2 shows the measured DDXs for all the angles from $0^{\circ}$ to $25^{\circ}$. Each neutron spectrum has a characteristic broad peak around $100 \mathrm{MeV}$ corresponding to half the incident energy, and the peak becomes sharper and sharper with decreasing angle. From this result, the neutron production is found to have very steep angular distribution. The broad peak can be explained by neutron emission from elastic and non-elastic deuteron breakup reactions. In addition, a weak peak is observed at the high energy end of each neutron spectrum at angles up to $10^{\circ}$. It is expected that this peak is formed by the transition to low-lying discrete levels in the residual nucleus by singleproton transfer reactions.

The experimental $0^{\circ}$-DDXs at $200 \mathrm{MeV}$ are compared with those measured previously at $25 \mathrm{MeV}$ [2], 40 $\mathrm{MeV}$ [3], and $102 \mathrm{MeV}$ [4] in Figure 3. Similarly, characteristic peaks around half the incident energy are clearly observed at all the low incident energies. As the incident energy increases, the peak position is shifted into higher energy, and the peak yield increases gradually up to 102 $\mathrm{MeV}$ and then decreases slightly at $200 \mathrm{MeV}$. Figure 4 shows the experimental differential cross sections (DXs) at $0^{\circ}$ for the $\operatorname{Li}(d, x n)$ and $\operatorname{Li}(p, n)$ reactions. The DXs were derived by integrating the DDXs around the observed broad peak shown in Figure 3, while those of the ${ }^{7} \operatorname{Li}(p, n)$ ${ }^{7}$ Be reaction measured in Refs. [10-13] include both transitions to the ground and the first excited states of the residual nucleus. It is found that neutron production in the $\mathrm{Li}(d, x n)$ reaction is larger than that in the ${ }^{7} \mathrm{Li}(p, n){ }^{7} \mathrm{Be}$ reaction by more than an order of magnitude. As for the incident energy dependence, the DXs of the $\operatorname{Li}(d, x n)$ reaction increase gradually with increasing incident energy, 
while the DXs of the ${ }^{7} \operatorname{Li}(p, n){ }^{7} \mathrm{Be}$ reaction has almost the constant (about $36 \mathrm{mb} / \mathrm{sr}$ ) at incident proton energies between 20 to $200 \mathrm{MeV}$.

\subsection{Comparison with model calculation and nuclear data}

In Figure 5, the measured DDXs are compared with model calculations with PHITS [5] and DEURACS [6] as well as TENDL-2017 nuclear data [7].

The PHITS code is based on a combination of different models to describe the total reaction cross section, the dynamical and subsequent evaporation processes. In the present calculation, the MWO model [14] is chosen for calculation of total reaction cross sections. INCL4.6 [15] and the generalized evaporation model (GEM) [16] are employed in simulating the dynamical process including deuteron breakup reaction and the subsequent evaporation process, respectively. In addition, we have used a new approach combining INCL and DWBA developed by Hashimoto et al. [17]. In DEURACS [6], elastic and nonelastic breakup reactions are described by the continuum discretized coupled channel theory and the Glauber model, respectively. In addition, DWBA is employed for singleproton transfer reactions to bound states in the residual nuclei.

As shown by the green dashed lines in Figure 5, the PHITS calculation is in overall agreement with the measured DDXs except in the low energy region where the evaporation process described by GEM is dominant and around the high energy end. In addition, the PHITS calculation underestimates the cross section at the broad peak seen around $100 \mathrm{MeV}$. On the other hand, the DEURACS calculation denoted by the red solid lines reproduces the measured DDXs, particularly around the broad peak, but underestimation is seen at neutron energies between 30 and $60 \mathrm{MeV}$. The peaks appearing near the high energy end in the DEURACS calculation correspond to the singleproton transfer reaction on ${ }^{7} \mathrm{Li}$ leading to the ground and the first excited states in the residual ${ }^{8} \mathrm{Be}$ nucleus.

In Figure 5, a comparison is also shown of the measured DDXs with TENDL-2017 data denoted by the blue dotted lines. Two broad peaks are seen in TENDL-2017 data. The evaporation peaks in the low neutron energy region overestimate the measured data remarkably. The broad peaks around $140 \mathrm{MeV}$ correspond to deuteron breakup component, and the position is shifted to higher neutron energy than the experimental peak seen around $100 \mathrm{MeV}$. The steep angular distribution of the broad peak around $100 \mathrm{MeV}$ is not reproduced by TENDL-2017 data at all.

Finally, the present benchmark test of theoretical models and nuclear data has demonstrated that DEURACS provides the best agreement with the measured DDXs. It should be noted that DEURACS calculations for lower incident energies $(25,40$, and $102 \mathrm{MeV})$ also show good agreement with the experimental data. Thus, we have compiled DDXs calculated by DEURACS for light particles and gamma-rays produced by the deuteron-induced reaction on ${ }^{7} \mathrm{Li}$ at incident energies up to $200 \mathrm{MeV}$ in the

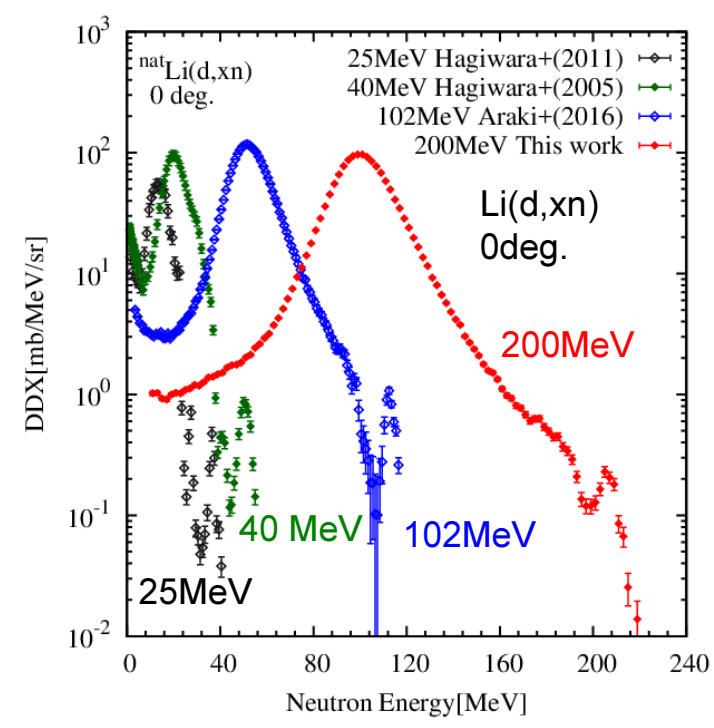

Figure 3. Measured double differential cross sections for the $\mathrm{Li}(d, x n)$ reactions at $25 \mathrm{MeV}$ [2], $40 \mathrm{MeV}$ [3], $102 \mathrm{MeV}$ [4], and $200 \mathrm{MeV}$.

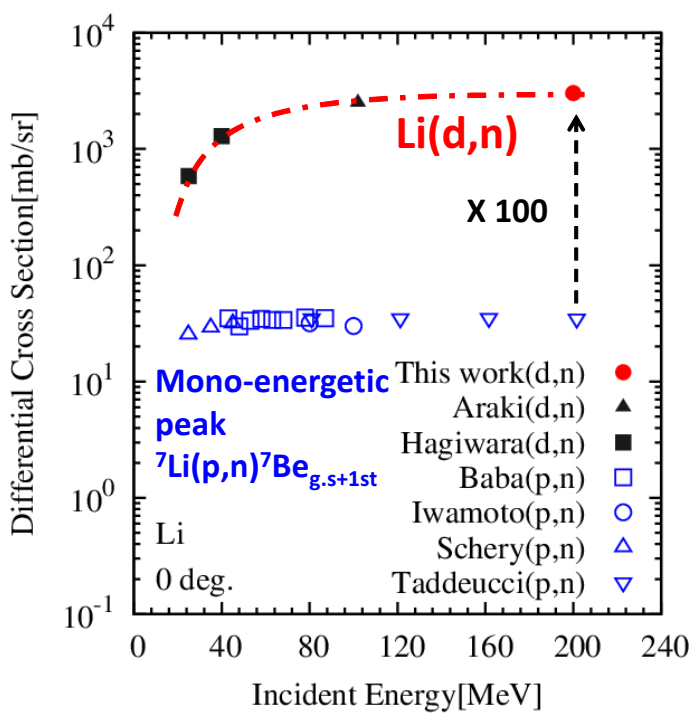

Figure 4. Measured differential cross sections at $0^{\circ}$ for the $\mathrm{Li}(d, x n)$ reaction and the ${ }^{7} \mathrm{Li}(p, n)^{7} \mathrm{Be}_{\text {g.s }+1 \text { st }}$ reaction at incident energies between $25 \mathrm{MeV}$ to $200 \mathrm{MeV}$.

Frag Data format uniquely defined in PHITS. The DDX database was applied to PHITS calculation of thick-target neutron yields (TTNYs) from deuteron bombardment on lithium at $40 \mathrm{MeV}$. The result is shown in Ref. [18]. The PHITS calculation with the DDX database reproduced the experimental TTNY better than the past PHITS calculation with INCL and GEM models.

\section{Summary and outlook}

We have measured double-differential cross sections for $200-\mathrm{MeV}(d, x n)$ reaction on $\mathrm{Li}$ at the emission angles ranging from $0^{\circ}$ to $25^{\circ}$ in steps of $5^{\circ}$. The incident energy 


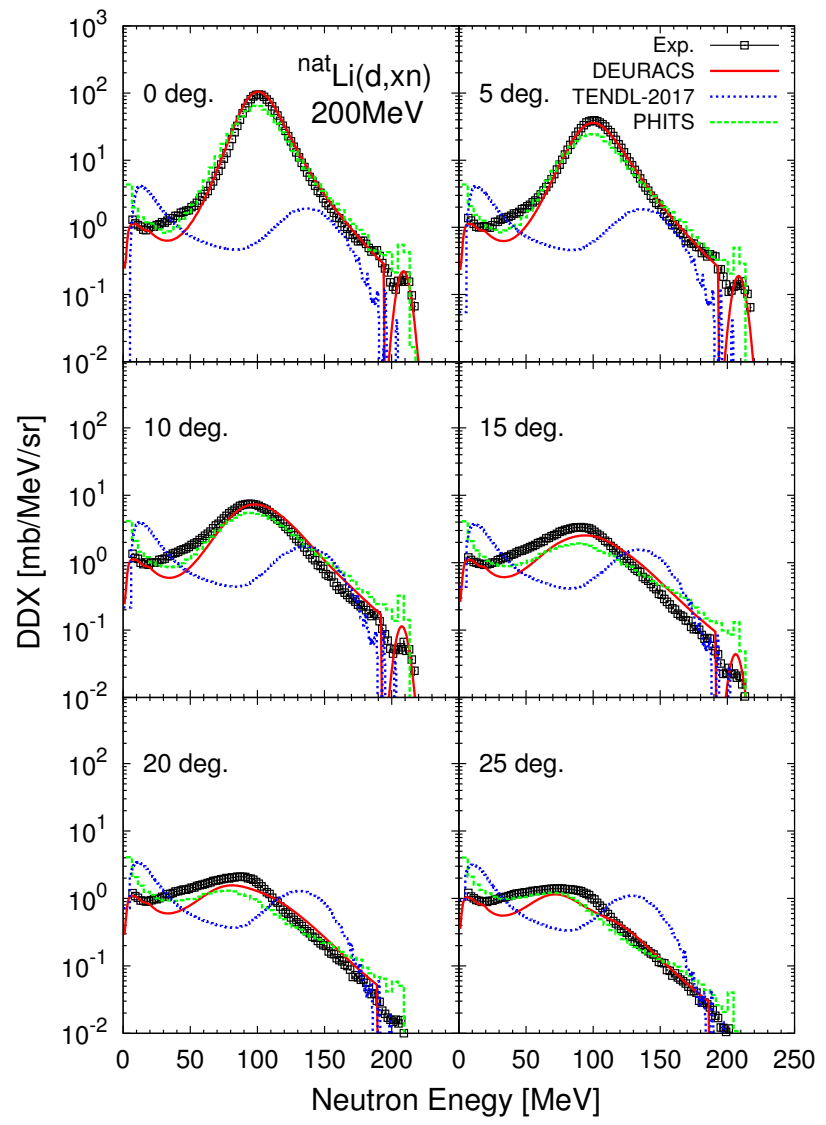

Figure 5. Comparison of measured DDXs for $\mathrm{Li}$ at emission angles from $0^{\circ}$ to $25^{\circ}$ with DEURACS and PHITS calculations, and TENDL-2017 nuclear data.

dependence of the neutron production was investigated in comparison with the $\mathrm{Li}(p, n)$ reaction up to $200 \mathrm{MeV}$. The measured data were compared with the model calculations with PHITS and DEURACS and the TENDL-2017 nuclear data. The DEURACS provided the best agreement with the experimental DDXs for all angles.

In the future, we plan to develop an evaluated deuteron nuclear data for Li stored in the ENDF format and the ACE file, and extend the present measurement of Li to the other targets of neutron converter materials (Be, $\mathrm{C}$, etc) and structure materials $(\mathrm{Al}, \mathrm{Cu}$, etc $)$.

\section{Acknowledgment}

This work was partially supported by ImPACT Program of Council for Science, Technology and Innovation (Cabinet Office, Government of Japan).

\section{References}

[1] EXFOR: https://www-nds.iaea.org/exfor/exfor.htm

[2] M. Hagiwara et al., J. of Nucl. Mater. 417, 1284 (2011)

[3] M. Hagiwara et al., Fusion Sci. and Technol. 48, 1320 (2005)

[4] S. Araki et al., Nucl. Inst. Meth. A 842, 62 (2017)

[5] T. Sato et al., J. Nucl. Sci. Technol. 55, 684 (2018)

[6] S. Nakayama et al., Phys. Rev. C 94, 014618 (2016); Phys. Rev. C 98, 044606 (2018); Phys. Rev. C 100, 044603 (2019).

[7] A. J. Koning et al., Nucl. Data Sheets 155, 1 (2019)

[8] D. Satoh et al., JAEA-Data/Code 2006-023, 1 (2006); J. of Nucl. Sci. and Technol. 43, 714 (2006)

[9] Y. Watanabe et al., AIP Conference Proc. 765, 326 (2005)

[10] Y. Iwamoto et al, Nucl. Instrum. Meth. A 80450 (2015)

[11] T. N. Taddeuchi et al, Nucl. Phys. A 469125 (1987)

[12] S. Schery et al, Nucl. Instrum. Meth. 147 399(1977)

[13] M. Baba et al, Nucl. Instrum. Meth. A 428 454(2000)

[14] K. Minomo et al., J. Nucl. Sci. Technol. 54, 127 (2017)

[15] A. Boudard et al., Phys. Rev. C 87014606 (2013)

[16] S. Furihata, Nucl. Instr. Meth. B 171, 251 (2000)

[17] S. Hashimoto et al., Nucl. Instr. Meth. B 333, 27 (2014)

[18] S. Nakayama et al., presented at this conference (2019) 